\title{
Diferencias sociodemográficas entre usuarios de inhalables, usuarios de otras drogas y adolescentes no consumidores en una muestra mexicana de estudiantes
}

\author{
María Elena Medina-Mora', Claudia Rafful², Jorge A. Villatoro Velázquez', Natania Oliva Robles', \\ Marycarmen Bustos Gamiño', Midiam Moreno' \\ ' Instituto Nacional de Psiquiatría Ramón de la Fuente Muñiz, México \\ ${ }^{2}$ División de Salud Global, Departamento de Medicina, Universidad de California en San Diego. Estados Unidos
}

\begin{abstract}
RESUMEN
Introducción: los inhalables como el pegamento, el thinner y otros derivados del petróleo son uno de los grupos de sustancias más utilizados entre la población adolescente mexicana. Estudios recientes han mostrado que, junto con la mariguana, los inhalables son el tipo de droga de preferencia de la población joven. Objetivo: el propósito de este estudio es determinar los factores que diferencian a los usuarios de inhalables de los usuarios de otras drogas, así como de quienes no las consumen. Método: se utilizaron datos de la Encuesta de Estudiantes 2009 del Distrito Federal, la cual contó con la participación de 22 mil 980 estudiantes de secundaria y preparatoria.

Resultados: los usuarios de inhalables son más jóvenes, provienen de niveles con menos recursos y sus padres y madres tienen menor nivel de escolaridad que los padres de los no usuarios; encontramos además que los usuarios de inhalables provienen de lugares en los que se tolera más el consumo de drogas, incluyendo el entorno familiar y social; son importantes también la disponibilidad y la oportunidad de consumo. En comparación con quienes consumen otras drogas, los usuarios de inhalables son más impulsivos, tienen más amigos con problemas antisociales y, con más frecuencia, reportan algún familiar usuario de drogas. Discusión y conclusiones: nuestros resultados reafirman la necesidad de diseñar programas de prevención que no se enfoquen únicamente en el consumo de drogas, sino en los factores familiares y sociales relacionados con un mayor riesgo de consumo.
\end{abstract}

\begin{abstract}
Introduction: inhalants like glue, paint thinner, and other petroleum-derived substances are one of the most utilized substance groups among Mexican teenagers. Recent studies have found that, along with marijuana, inhalants are the preferred substance of abuse among youth. Objective: the purpose of the present study is to determine factors that differentiate inhalant users from other drug users and those who do not consume any drugs. Method: data from the 2009 Student Survey for Mexico City was used, which consisted a sample of 22,980 middle and high school students. Results: inhalant users are younger, come from locations in which drug use is more tolerated, including family and social enviroments; availability and opportunity for use were also important. In comparison with other drug users, inhalant users are more impulsive, have more friends with antisocial behaviors and had with more frequency a family member who is also a drug user. Discussion and conclusions: results reassert the need for designing prevention programs that not only focus on drug use, but are also aimed at family and social factors related to higher risk of use.
\end{abstract}

Key Words: inhalant; risk factors; students; Mexico.

Palabras clave: inhalables; factores de riesgo; estudiantes; México.

\footnotetext{
Autora de correspondencia:

María Elena Medina-Mora. Dirección General, Instituto Nacional de Psiquiatría Ramón de la Fuente Muñiz. Calzada México Xochimilco núm. 101, col. San Lorenzo Huipulco, del. Tlalpan, C.P. 14370, Distrito Federal, México. Tel.: +52(55) 56550185. Correo electrónico: medinam@imp.edu.mx.

Recibido: 01 de agosto del 2015.

Aceptado: 18 de septiembre del 2015.

DOI: 10.28931/riiad.2015.1.02
} 


\section{INTRODUCCIÓN}

El uso de inhalables o solventes incluye una lista prácticamente interminable de sustancias volátiles autoadministradas que no han sido producidas para el consumo humano pero que, si se inhalan, producen efectos psicoactivos (Balster, Cruz, Howard, Dell, \& Cottler, 2008; Cruz, 2011). Entre los inhalables que se consumen con mayor frecuencia por la población mexicana se encuentran el tolueno, la pintura, el pegamento y el thinner (Balster et al., 2008; Cruz, 2011).

Los inhalables son considerados como el tipo de sustancias de abuso que mayor daño causan al organismo, incluyendo enfermedades respiratorias y deficiencias cognitivas (Maclver, 2009; Rosenberg, Spitz, Filley, Davis, \& Schaumburg, 1988; Toga, Thompson, \& Sowell, 2006; Yucel, Takagi, Walterfang, \& Lubman, 2008; Yucel et al., 2010). En los países desarrollados, el uso de estas sustancias se asocia frecuentemente con una fase de experimentación que se da en la adolescencia (Degenhardt et al., 2008); sin embargo, debido a su carácter legal y bajo costo, su consumo en países en desarrollo se reporta entre poblaciones en condiciones de vulnerabilidad, sobre todo en niños, adolescentes, trabajadoras sexuales e indigentes (Gigengack, 2014b, 2014d; Morris et al., 2011; Villatoro, Cruz, Ortiz, \& Medina-Mora, 2011), entre otros.

La adolescencia es una etapa clave en el desarrollo cerebral, debido a que aún se están fortaleciendo y generando conexiones sinápticas en el área prefrontal y hay un incremento en la materia blanca (Toga et al., 2006). La adolescencia es también un periodo de búsqueda de sensaciones y experimentación con sustancias, incluidos los inhalables. En estudios longitudinales y transversales se ha encontrado que el consumo de estas drogas en edades tempranas predice el uso simultáneo de opiáceos, así como el desarrollo de trastorno por uso de opiáceos en la adultez (Schepis \& Krishnan-Sarin, 2008; Storr, Westergaard, \& Anthony, 2005; Wu, Schlenger, \& Ringwalt, 2005). Los cambios neuropsicológicos relacionados con el uso de inhalables son mediados por las características del contexto psicosocial, como la historia de consumo de drogas en la familia, un nivel socioeconómico bajo (Nonnemaker, Crankshaw, Shive, Hussin, \& Farrelly, 2011) y la comorbilidad con trastornos mentales (Takagi, Lubman, \& Yucel, 2007; Vaughn, Perron, \& Howard, 2007; Wu et al., 2005). Como pasa con otras drogas, pero en mayor medida, los adolescentes consideran el uso de inhalables como un medio de escape de la realidad, así como una herramienta para socializar (Siegel, Alvaro, Patel, \& Crano, 2009).

Los estudios realizados en México muestran que la edad de inicio de uso de solventes es de entre 12 y
14 años (Villatoro, Cruz et al., 2011). Para los 17 años, $77 \%$ de los consumidores ya los han usado al menos una vez en la vida (Instituto Nacional de Psiquiatría Ramón de la Fuente Muñiz [INPRF], Instituto Nacional de Salud Pública [INSP], \& Secretaría de Salud [SSA], 2012).

El uso de inhalables solía ser más común en grupos en condiciones de vulnerabilidad, como aquellos que trabajan o viven en las calles (Gigengack, 2014a, 2014c; Medina-Mora, Villatoro, Fleiz, Domínguez, \& Cruz, 2014), no obstante, en la actualidad el consumo de inhalables se ha incrementado entre los estudiantes que viven con sus familias (Villatoro, Cruz et al., 2011): los estudiantes entre primero de secundaria y tercero de preparatoria reportan prevalencias de uso alguna vez en la vida de $10.4 \%$ (INPRF et al., 2012). Sin embargo, de acuerdo con datos de estudios en poblaciones especiales (Gigengack, 2014a) y reportes de centros de tratamiento, esta prevalencia es más alta entre poblaciones en situación de vulnerabilidad que no se incluyen en las encuestas de población general, tal es el caso de los niños en situación de calle (Villatoro, Cruz et al., 2011). Los hombres reportan mayor consumo que las mujeres, pero esta brecha se ha ido cerrando en la población adulta general y entre los estudiantes (Villatoro, Cruz et al., 2011).

En la Encuesta de Estudiantes de la Ciudad de México de 2006 se reportó un incremento en el uso de inhalables en comparación con mediciones anteriores (Villatoro et al., 2009). Para 2009, la encuesta reportó que el mayor incremento en el consumo de drogas se dio en los inhalables, sustancias que para los hombres se convirtieron en la segunda droga de preferencia después de la mariguana $(7.7 \%$ y $10.1 \%$, respectivamente en el último año); en las mujeres, en cambio, fueron la primera droga de preferencia, seguida por la mariguana $(7.2 \%$ y $6.2 \%$, respectivamente en este mismo periodo) (Villatoro, Gaytán et al., 2011).

El propósito de este estudio es analizar, con información de dicha encuesta, los factores psicosociales relacionados con el uso de inhalables entre estudiantes de secundaria y preparatoria de la Ciudad de México.

\section{MÉTODO}

\section{Muestra}

La Encuesta de Estudiantes 2009 del Distrito Federal contó con la participación de 22 mil 980 estudiantes de secundaria y preparatoria, $49.8 \%$ mujeres y $50.2 \%$ hombres. El diseño de la muestra fue estratificado y por conglomerados; la unidad de selección fue el grupo escolar. 


\section{Participantes}

Los participantes en el estudio fueron aquellos estudiantes de secundaria y bachillerato inscritos en el ciclo escolar 2008-2009 que estuvieron presentes al momento de la aplicación. La selección del grupo se hizo a través de una selección aleatoria, en la que el encuestador tenía un número de grupo seleccionado; al llegar a la institución educativa, el aplicador realizaba un listado del total de grupos en la escuela con criterios específicos y observaba a qué grupo pertenecía el número seleccionado. Se requirió que estuviera presente $80 \%$ de los asistentes regulares del grupo para proceder con la aplicación del cuestionario. Se solicitó la participación voluntaria de cada alumno y se hizo hincapié en que las respuestas eran confidenciales y anónimas.

\section{Instrumento}

Se utilizó un cuestionario estandarizado autoadministrado que ha sido aplicado y validado en las Encuestas de Estudiantes anteriores tanto en la Ciudad de México como en otras entidades federativas. Los indicadores de consumo de drogas son comparables con los indicadores de los observatorios internacionales de Europa (Observatorio Europeo de las Drogas y las Toxicomanías [EMCDDA], 2015), así como con los usados en el estudio "Monitoring the Future" en Estados Unidos (Johnston, O'MaIley, Miech, Bachman, \& Schulenberg, 2015). El proyecto para el levantamiento de la encuesta fue aprobado por el Comité de Ética del Instituto Nacional de Psiquiatría Ramón de la Fuente Muñiz y por la Secretaría de Educación Pública. Los detalles del muestreo y de la Encuesta de Estudiantes 2009 se pueden encontrar en publicaciones previas (Villatoro, Gaytán et al., 2011). Asimismo, el cuestionario está disponible en http://www.uade.inpsiquiatria. edu.mx; los datos de las variables usadas para este estudio se encuentran en el siguiente apartado.

\section{Variables}

Para este trabajo se utilizaron distintas variables que se describen a continuación:

- Oportunidad de uso de drogas: ofrecimiento de drogas, sean regaladas o vendidas. Los valores son: ha tenido (1) o no oportunidad (0).

- Disponibilidad de drogas: facilidad con la que un alumno puede conseguir alguna sustancia ilícita. Sus valores son disponibles ( 1 = fácil y muy fácil), no disponibles (0).

- Impulsividad: realizar una respuesta rápida, sin valoración de por medio de las situaciones cotidianas que vive el adolescente. Variable continua con tres reactivos con calificación de 1 a 3 , donde un mayor valor indica mayor impulsividad.

- Satisfacción baja con la calidad de vida: evalúa el nivel de agrado que se tiene en diferentes áreas de su vida como salud física, relación con sus padres, lugar de residencia, etcétera. Es una variable continua, resultado de la sumatoria de ocho reactivos. Su escala es de 1 a 4 , donde un mayor valor indica un menor nivel de satisfacción

- Desajuste social: nivel de oposición o aceptación del alumno frente a las normas sociales aprendidas. Variable continua, resultado de la sumatoria de ocho reactivos, en una escala de 1 a 4 , donde un mayor valor indica un mayor desajuste hacia las normas sociales.

- Autoestima: pensamientos y sentimientos positivos y negativos en referencia a sí mismo. Variable continua con valores de 1 a 4 . Un mayor valor indica menor autoestima.

- Involucramiento parental: interés de los padres por los gustos y actividades de sus hijos o hijas. Variable continua con valores de 1 a 4 , donde un mayor valor indica un menor involucramiento.

- Monitoreo negativo: conocimiento de los padres de las actividades y relaciones de sus hijos o hijas para verificar qué hacen, con quién o dónde se encuentran. Variable continua con valores entre 1 y 4 , donde un mayor valor indica un mayor monitoreo negativo.

- Aliento parental: relación afectuosa que los padres mantienen con sus hijos o hijas para motivarlos en todas sus actividades o, en su caso, halagarlos cuando hacen las cosas bien. Variable continua con valores entre 1 y 4 , donde un mayor valor indica un menor aliento parental.

- Disciplina inconsistente: uso de amenazas verbales o consecuencias que los padres dirigen hacia sus hijos o hijas, cuya finalidad es mantener la disciplina al interior de la familia, pero que no son cumplidas por los padres. Variable continua con valores entre 1 y 4 , donde un mayor valor indica mayor inconsistencia en la disciplina.

- Relación con pares: esta escala incluye reactivos que permiten conocer información relativa a las conductas y prácticas que realiza el grupo de amigos más cercano al alumno. Consta de dos escalas con valores que van de 1 a 4 . Una de las escalas evalúa la presencia de amigos antisociales y la otra de amigos prosociales. En ambos casos se utilizan variables continuas, donde a mayor valor, mayor presencia de pares antisociales, en un caso; mientras que en el otro, un mayor valor indica menor presencia de pares prosociales.

- Percepción del riesgo de uso de drogas ilícitas: grado de peligrosidad que el alumno atribuye a las consecuencias negativas de consumir sustancias médi- 
cas o ilegales. Variable dicotómica, donde los valores son: (1) baja percepción de riesgo y (0) sí se percibe riesgo en el consumo.

- Percepción del riesgo de uso de drogas legales: grado de peligrosidad que el alumno atribuye a las consecuencias negativas de consumir alcohol o tabaco. Variable dicotómica, donde los valores son: (1) baja percepción de riesgo y (0) sí se percibe riesgo.

\section{Análisis estadístico}

Se hicieron análisis descriptivos de las características personales, sociodemográficas y del ambiente. Además de incluir edad y nivel socioeconómico, como en otras investigaciones, también se contemplaron características como el estatus académico y laboral, la asertividad, la impulsividad, la calidad de vida, la autoestima, el tipo de parentalidad y las horas pasadas con los padres, entre otras. En las Tablas 1, 2, 3 y 4 se presentan dichas características para cada uno de los tres grupos de análisis (no usuarios, usuarios de inhalables y usuarios de otras drogas) por sexo.
Debido a que el principal objetivo es diferenciar a los usuarios de inhalables del resto de los estudiantes, se hicieron dos análisis de regresiones multivariadas: el primero, para comparar a éstos con quienes no usan drogas, y el segundo para comparar a los usuarios de inhalables con los consumidores de otras drogas. Para ello, se determinó la razón de momios (RM), tomando ambas veces como referencia a los que no usaron inhalables. Se utilizó el paquete estadístico STATA versión 11.0 para muestras complejas, con el número de grupo como unidad de muestreo.

\section{RESULTADOS}

\section{Resultados bivariados}

Características personales por tipo de droga y sexo

Menos de $10 \%$ ( $9.7 \%$ en hombres y $9.5 \%$ en mujeres) de los estudiantes de secundaria, y $12.1 \%$ de los hombres y $10.7 \%$ de las mujeres que estudian preparatoria,

Tabla 1

Características personales por tipo de droga y sexo

\begin{tabular}{|c|c|c|c|c|c|c|c|c|c|c|c|c|}
\hline & \multicolumn{6}{|c|}{ Hombres } & \multicolumn{6}{|c|}{ Mujeres } \\
\hline & \multicolumn{2}{|c|}{ No uso } & \multicolumn{2}{|c|}{$\begin{array}{c}\text { Uso de } \\
\text { inhalables }\end{array}$} & \multicolumn{2}{|c|}{$\begin{array}{c}\text { Uso de otras } \\
\text { drogas }\end{array}$} & \multicolumn{2}{|c|}{ No uso } & \multicolumn{2}{|c|}{$\begin{array}{c}\text { Uso de } \\
\text { inhalables }\end{array}$} & \multicolumn{2}{|c|}{$\begin{array}{c}\text { Uso de otras } \\
\text { drogas }\end{array}$} \\
\hline & $n$ & $\%$ & $n$ & $\%$ & $n$ & $\%$ & $n$ & $\%$ & $n$ & $\%$ & $\mathrm{n}$ & $\%$ \\
\hline \multicolumn{13}{|l|}{ Nivel académico } \\
\hline Secundaria & 182462 & 83.6 & 21220 & 9.7 & 14488 & 6.6 & 182396 & 84.1 & 20553 & 9.5 & 13856 & 6.4 \\
\hline Preparatoria & 118240 & 69.5 & 20562 & 12.1 & 31357 & 18.4 & 123530 & 73.6 & 17909 & 10.7 & 26403 & 15.7 \\
\hline \multicolumn{13}{|c|}{ Estatus académico } \\
\hline $\begin{array}{l}\text { Estudiante de } \\
\text { medio tiempo }\end{array}$ & 53412 & 68.2 & 11166 & 14.3 & 13736 & 17.5 & 39746 & 70.1 & 8524 & 15.0 & 8450 & 14.9 \\
\hline $\begin{array}{l}\text { Estudiante de } \\
\text { tiempo } \\
\text { completo }\end{array}$ & 244123 & 79.9 & 29964 & 9.8 & 31424 & 10.3 & 261078 & 81.3 & 28879 & 9.0 & 31250 & 9.7 \\
\hline \multicolumn{13}{|l|}{ Estatus laboral } \\
\hline Desempleado & 237350 & 81.2 & 26313 & 9.0 & 28780 & 9.8 & 271403 & 81.3 & 30331 & 9.1 & 32255 & 9.7 \\
\hline Empleado & 58221 & 65.4 & 14738 & 16.6 & 16025 & 18.0 & 30070 & 66.7 & 7453 & 16.5 & 7576 & 16.8 \\
\hline \multicolumn{13}{|c|}{ Nivel socioeconómico } \\
\hline Bajo & 37796 & 73.6 & 7117 & 13.9 & 6445 & 12.5 & 54173 & 77.5 & 8605 & 12.3 & 7126 & 10.2 \\
\hline Medio-bajo & 54637 & 77.6 & 7708 & 10.9 & 8084 & 11.5 & 60227 & 79.1 & 8331 & 10.9 & 7580 & 10.0 \\
\hline Medio & 61794 & 79.9 & 7839 & 10.1 & 7658 & 9.9 & 58409 & 80.8 & 6450 & 8.9 & 7409 & 10.3 \\
\hline Medio-alto & 49596 & 80.1 & 5251 & 8.5 & 7097 & 11.5 & 42673 & 80.9 & 4861 & 9.2 & 5195 & 9.9 \\
\hline Alto & 72255 & 76.3 & 9640 & 10.2 & 12865 & 13.6 & 65522 & 80.2 & 6286 & 7.7 & 9933 & 12.2 \\
\hline \multicolumn{13}{|l|}{ Abuso sexual } \\
\hline No & 280123 & 79.0 & 34614 & 9.8 & 39696 & 11.2 & 287539 & 81.8 & 30735 & 8.7 & 33251 & 9.5 \\
\hline Sí & 20579 & 60.7 & 7167 & 21.1 & 6149 & 18.1 & 18387 & 55.5 & 7727 & 23.3 & 7008 & 21.2 \\
\hline
\end{tabular}


Características personales por tipo de droga y sexo

\begin{tabular}{|c|c|c|c|c|c|c|c|c|c|c|c|c|}
\hline & \multicolumn{6}{|c|}{ Hombres } & \multicolumn{6}{|c|}{ Mujeres } \\
\hline & \multicolumn{2}{|c|}{ No usuario } & \multicolumn{2}{|c|}{$\begin{array}{l}\text { Usuario de } \\
\text { inhalables }\end{array}$} & \multicolumn{2}{|c|}{$\begin{array}{l}\text { Usuario de } \\
\text { otras drogas }\end{array}$} & \multicolumn{2}{|c|}{ No usuario } & \multicolumn{2}{|c|}{$\begin{array}{l}\text { Usuario de } \\
\text { inhalables }\end{array}$} & \multicolumn{2}{|c|}{$\begin{array}{l}\text { Usuario de } \\
\text { otras drogas }\end{array}$} \\
\hline & Media & $\mathrm{DE}$ & Media & $\mathrm{DE}$ & Media & $\mathrm{DE}$ & Media & $\mathrm{DE}$ & Media & $\mathrm{DE}$ & Media & $\mathrm{DE}$ \\
\hline Impulsividad & 1.5 & 0.6 & 1.8 & 0.6 & 1.7 & 0.6 & 1.4 & 0.5 & 1.8 & 0.6 & 1.6 & 0.5 \\
\hline $\begin{array}{l}\text { Satisfacción con la } \\
\text { calidad de vida }\end{array}$ & 1.9 & 1.0 & 2.1 & 0.9 & 2.1 & 0.9 & 2.0 & 0.9 & 2.3 & 0.8 & 2.3 & 0.8 \\
\hline Ajuste social & 2.7 & 0.4 & 2.4 & 0.5 & 2.5 & 0.4 & 2.7 & 0.3 & 2.4 & 0.5 & 2.5 & 0.4 \\
\hline Autoestima & 3.3 & 0.8 & 3.1 & 0.8 & 3.1 & 0.9 & 3.2 & 0.7 & 3.0 & 0.8 & 3.1 & 0.8 \\
\hline
\end{tabular}

han usado inhalables alguna vez en la vida (Tablas 1 y 2). Esta proporción es mayor de la que se observa para otras drogas en secundaria $(6.6 \%$ y $6.4 \%$ en hombres y mujeres) y menor de la que se reporta en preparatoria (18.4\% y $15.7 \%$, respectivamente). Entre los usuarios de drogas de secundaria, poco más de la mitad, 60\%, ha usado inhalables; en la preparatoria, la proporción disminuye a $39 \%$ de los hombres usuarios y a $28 \%$ de las mujeres que han usado una o más drogas alguna vez en su vida.

Los participantes que dijeron haber sido estudiantes de medio tiempo en el último año reportaron mayor proporción de consumo de inhalables que los estudiantes de tiempo completo. Esta diferencia tiende a ser mayor en las mujeres (15\% vs. 9.0\%) que en los hombres (14.3\% vs. 9.8\%). Asimismo, quienes afirmaron haber trabajado en el último año reportaron mayor uso que los que señalaron no haberlo hecho; dicha tendencia fue similar en hombres y mujeres. Resalta que una mayor proporción de las personas de nivel socioeconómico bajo (tanto hombres como mujeres) reportó consumo de inhalables, superando así al uso del resto de las drogas. En contraste, las personas con nivel socioeconómico alto reportaron mayor consumo de otras drogas que de inhalables.

En cuanto a abuso sexual, de las personas que afirmaron haber sufrido esta experiencia, $21.1 \%$ de los hombres y $23.3 \%$ de las mujeres señalaron el uso de inhalables, el cual es mayor, en ambos casos, al consumo de otras drogas (18.1\% y 21.2, respectivamente).

\section{Características sociales y familiares por tipo de droga y sexo}

Dentro de los dos grupos de usuarios de drogas, los consumidores de inhalables fueron los que reportaron un nivel educativo menor de sus padres (Tablas 3 y 4 ).
En cuanto a las horas pasadas con los padres, tanto en hombres como en mujeres la menor proporción de usuarios se ubica entre quienes reportaron pasar más tiempo con el padre o con la madre. Es más frecuente que los estudiantes del sexo masculino que tienen parientes con problemas de consumo de alcohol usen inhalables (14.2\%), en comparación con quienes no están en esta situación (8.6\%); en mujeres, las diferencias entre quienes tienen o no tienen familiares con este problema son menores (11.5\% y $9 \%$, respectivamente). El tener familiares que usan drogas tiene más influencia y afecta igual a hombres y mujeres, 23\% de los estudiantes, hombres y mujeres, con antecedentes de consumo en la familia reportó usar inhalables, mientras que los porcentajes para el consumo de otras drogas fueron 22 y $21 \%$, respectivamente. Las peleas entre los adultos que viven en su hogar son reportadas con más frecuencia entre quienes abusan de inhalables que entre los usuarios de otras sustancias. Otras variables que influyen en el consumo de inhalables y de otras drogas en hombres y mujeres son el tener amigos que usan drogas, el percibir que es fácil obtenerlas o el haber tenido una oportunidad para probarlas. Cabe resaltar que la mitad de los estudiantes que señalan haber tenido la oportunidad de usar drogas las han consumido; en contraste, sólo 10\% de los que dicen no haber tenido la oportunidad, es decir, que les hayan regalado o vendido drogas, reporta haberlas usado (Tabla 3).

\section{Resultados de las regresiones}

En la Tabla 5 se presentan los resultados de las regresiones multivariadas. Se compara a los consumidores de inhalables con los no usuarios y a los usuarios de inhalables con los de otras drogas, por separado para hombres y mujeres. 
Tabla 3

Características sociales y familiares por tipo de drogas usadas y sexo

\begin{tabular}{|c|c|c|c|c|c|c|c|c|c|c|c|c|}
\hline & \multicolumn{6}{|c|}{ Hombres } & \multicolumn{6}{|c|}{ Mujeres } \\
\hline & \multicolumn{2}{|c|}{ No usa } & \multicolumn{2}{|c|}{$\begin{array}{c}\text { Uso de } \\
\text { inhalables }\end{array}$} & \multicolumn{2}{|c|}{$\begin{array}{c}\text { Uso de otras } \\
\text { drogas }\end{array}$} & \multicolumn{2}{|c|}{ No usa } & \multicolumn{2}{|c|}{$\begin{array}{c}\text { Uso de } \\
\text { inhalables }\end{array}$} & \multicolumn{2}{|c|}{$\begin{array}{c}\text { Uso de otras } \\
\text { drogas }\end{array}$} \\
\hline & $n$ & $\%$ & $n$ & $\%$ & $n$ & $\%$ & $n$ & $\%$ & $n$ & $\%$ & $n$ & $\%$ \\
\hline \multicolumn{13}{|l|}{$\begin{array}{l}\text { Educación del } \\
\text { padre }\end{array}$} \\
\hline $\begin{array}{l}\text { Secundaria o } \\
\text { menos }\end{array}$ & 121108 & 75.8 & 20733 & 13.0 & 17932 & 11.2 & 134367 & 79.5 & 20136 & 11.9 & 14514 & 8.6 \\
\hline $\begin{array}{l}\text { Preparatoria o } \\
\text { más }\end{array}$ & 156281 & 78.1 & 18669 & 9.3 & 25157 & 12.6 & 147647 & 79.1 & 15516 & 8.3 & 23433 & 12.6 \\
\hline \multicolumn{13}{|l|}{$\begin{array}{l}\text { Educación de la } \\
\text { madre }\end{array}$} \\
\hline $\begin{array}{l}\text { Secundaria o } \\
\text { menos }\end{array}$ & 134069 & 76.4 & 22026 & 12.6 & 19409 & 11.1 & 147153 & 79.6 & 21876 & 11.8 & 15918 & 8.6 \\
\hline $\begin{array}{l}\text { Preparatoria o } \\
\text { más }\end{array}$ & 148919 & 77.8 & 17906 & 9.4 & 24674 & 12.9 & 143356 & 79.2 & 14976 & 8.3 & 22697 & 12.5 \\
\hline \multicolumn{13}{|l|}{ Horas pasadas } \\
\hline $5+$ & 112553 & 82.4 & 12074 & 8.8 & 11929 & 8.7 & 113237 & 84.3 & 10894 & 8.1 & 10216 & 7.6 \\
\hline $3-4$ & 80150 & 78.3 & 10178 & 9.9 & 12065 & 11.8 & 70092 & 80.2 & 8297 & 9.5 & 9020 & 10.3 \\
\hline $1-2$ & 39396 & 74.1 & 5980 & 11.3 & 7757 & 14.6 & 41406 & 75.7 & 5878 & 10.8 & 7386 & 13.5 \\
\hline Ninguna & 64956 & 71.6 & 12693 & 14.0 & 13058 & 14.4 & 77961 & 75.0 & 12929 & 12.4 & 13013 & 12.5 \\
\hline \multicolumn{13}{|l|}{$\begin{array}{l}\text { Horas pasadas } \\
\text { con la madre }\end{array}$} \\
\hline $5+$ & 208837 & 80.6 & 24381 & 9.4 & 26023 & 10.0 & 232004 & 82.5 & 24340 & 8.7 & 24905 & 8.9 \\
\hline $3-4$ & 48969 & 72.3 & 8238 & 12.2 & 10562 & 15.6 & 39377 & 73.2 & 7004 & 13.0 & 7381 & 13.7 \\
\hline $1-2$ & 19010 & 67.0 & 4404 & 15.5 & 4962 & 17.5 & 13561 & 67.7 & 2732 & 13.6 & 3752 & 18.7 \\
\hline Ninguna & 21516 & 71.6 & 4481 & 14.9 & 4036 & 13.4 & 19859 & 70.4 & 4313 & 15.3 & 4044 & 14.3 \\
\hline \multicolumn{13}{|c|}{$\begin{array}{l}\text { Uno o más parientes } \\
\text { con problemas por } \\
\text { uso de alcohol }\end{array}$} \\
\hline No & 190705 & 80.3 & 20406 & 8.6 & 26251 & 11.1 & 190148 & 81.8 & 21026 & 9.0 & 21245 & 9.1 \\
\hline Sí & 109997 & 72.9 & 21375 & 14.2 & 19594 & 13.0 & 115778 & 76.1 & 17436 & 11.5 & 19014 & 12.5 \\
\hline \multicolumn{13}{|c|}{$\begin{array}{l}\text { Peleas entre adultos } \\
\text { en el hogar }\end{array}$} \\
\hline No & 252215 & 79.1 & 30283 & 9.5 & 36451 & 11.4 & 226713 & 83.5 & 20381 & 7.5 & 24416 & 9.0 \\
\hline Sí & 48487 & 69.9 & 11498 & 16.6 & 9394 & 13.5 & 79213 & 70.0 & 18081 & 16.0 & 15844 & 14.0 \\
\hline \multicolumn{13}{|l|}{$\begin{array}{l}\text { Uso de drogas en } \\
\text { la familia }\end{array}$} \\
\hline No & 281664 & 79.8 & 33404 & 9.5 & 37970 & 10.8 & 284133 & 82.3 & 29104 & 8.4 & 32080 & 9.3 \\
\hline Sí & 19038 & 53.9 & 8378 & 23.7 & 7875 & 22.3 & 21793 & 55.4 & 9358 & 23.8 & 8180 & 20.8 \\
\hline \multicolumn{13}{|l|}{$\begin{array}{l}\text { Amigos aceptan el } \\
\text { uso de drogas }\end{array}$} \\
\hline No & 256214 & 82.2 & 25059 & 8.0 & 30483 & 9.8 & 277587 & 83.6 & 25483 & 7.7 & 29107 & 8.8 \\
\hline Sí & 44488 & 58.1 & 16722 & 21.8 & 15362 & 20.1 & 28339 & 54.0 & 12980 & 24.7 & 11152 & 21.3 \\
\hline \multicolumn{13}{|l|}{$\begin{array}{l}\text { Mejor amigo usa } \\
\text { drogas }\end{array}$} \\
\hline No & 258271 & 84.4 & 20482 & 6.7 & 27078 & 8.9 & 263484 & 86.6 & 18814 & 6.2 & 21856 & 7.2 \\
\hline Sí & 42431 & 51.4 & 21299 & 25.8 & 18767 & 22.7 & 42442 & 52.7 & 19648 & 24.4 & 18403 & 22.9 \\
\hline \multicolumn{13}{|l|}{$\begin{array}{l}\text { Oportunidad de } \\
\text { uso de drogas }\end{array}$} \\
\hline No & 234816 & 90.9 & 11916 & 4.6 & 11656 & 4.5 & 255986 & 90.3 & 13713 & 4.8 & 13776 & 4.9 \\
\hline Sí & 65886 & 50.7 & 29866 & 23.0 & 34189 & 26.3 & 49940 & 49.4 & 24750 & 24.5 & 26483 & 26.2 \\
\hline $\begin{array}{l}\text { Disponibilidad de } \\
\text { drogas }\end{array}$ & & & & & & & & & & & & \\
\hline Acceso difícil & 199551 & 87.6 & 11532 & 5.1 & 16664 & 7.3 & 215376 & 88.1 & 13268 & 5.4 & 15827 & 6.5 \\
\hline Acceso fácil & 97658 & 62.6 & 29754 & 19.1 & 28702 & 18.4 & 87970 & 64.4 & 24520 & 18.0 & 24038 & 17.6 \\
\hline
\end{tabular}


No usuarios vs. usuarios de inhalables

- Diferencias sociodemográficas: los hombres usuarios de inhalables tienen una razón de momios significativamente mayor que los no consumidores de drogas de ser estudiantes de secundaria más que de preparatoria, tener un padre con escolaridad menor (secundaria o menos) y haber trabajado en el último año. En las mujeres, cursar un nivel menor (secundaria) aumenta la probabilidad de inhalar, pero la escolaridad del papá o el haber trabajado no ejercen una influencia significativa.

- Disponibilidad de drogas: las personas que han usado inhalables tienen mayor oportunidad de consumo de cualquier droga, así como una baja percepción de riesgo del uso de sustancias; asimismo, reportan tener un acceso fácil a las drogas, señalan, con más frecuencia, que sus familiares y mejor amigo son usuarios de drogas y viven en un ambiente en el que los pares toleran el uso de sustancias. La oportunidad de consumo es la variable que más incrementa el riesgo de inhalar.

- Diferencias psicológicas: los consumidores de inhalables, en comparación con los no usuarios, tienen mayor impulsividad y mayor desajuste social.

- Variables interpersonales: la familia y los amigos juegan un papel importante en la decisión del estudiante de usar inhalables; sin embargo, hay diferencias importantes entre ellos. En los hombres, el monitoreo negativo de los padres y el tener amigos con conductas antisociales aumentan el riesgo de consumir inhalables; en tanto que en las mujeres, convivir con sus madres menos de cinco horas a la semana y que los adultos peleen en sus casas, se convierten en importantes factores de riesgo.

Usuarios de inhalables vs. usuarios de otras drogas

Al comparar a los hombres consumidores de inhalables con los usuarios de otras drogas, las diferencias se reducen, pero se mantiene significativamente mayor el riesgo de uso de inhalables entre aquellos que tienen padres con escolaridad menor o igual a secundaria, están cursando la educación media, reportan tener oportunidad de consumo de drogas, tienen una baja percepción de riesgo de drogas ilegales, pueden acceder de manera fácil a las sustancias, presentan desajuste social y tienen un mejor amigo consumidor.

En el caso de las mujeres, las diferencias significativas entre las usuarias de inhalables y de otras drogas son: estar cursando la secundaria, que el padre tenga estudios de secundaria o menos, presentar impulsividad, tener una baja percepción de riesgo de las sustancias ilegales, percibir un acceso fácil a las drogas y convivir sólo entre una y dos horas con el padre. Tener pocos amigos con conductas prosociales disminuye la razón de uso de inhalables (Tabla 5).

\section{DISCUSIÓN Y CONCLUSIONES}

En la Ciudad de México, en una muestra de estudiantes, los adolescentes que han consumido inhalables muestran ciertas características distintas a las de los otros adolescentes. En general, los usuarios de estas sustancias son primordialmente estudiantes de secun-

Tabla 4

Características sociales y familiares por tipo de drogas usadas y sexo

\begin{tabular}{|c|c|c|c|c|c|c|c|c|c|c|c|c|}
\hline & \multicolumn{6}{|c|}{ Hombres } & \multicolumn{6}{|c|}{ Mujeres } \\
\hline & \multicolumn{2}{|c|}{ No usuario } & \multicolumn{2}{|c|}{$\begin{array}{l}\text { Usuario de } \\
\text { inhalables }\end{array}$} & \multicolumn{2}{|c|}{$\begin{array}{l}\text { Usuario de } \\
\text { otras drogas }\end{array}$} & \multicolumn{2}{|c|}{ No usuario } & \multicolumn{2}{|c|}{$\begin{array}{l}\text { Usuario de } \\
\text { inhalables }\end{array}$} & \multicolumn{2}{|c|}{$\begin{array}{l}\text { Usuario de } \\
\text { otras drogas }\end{array}$} \\
\hline & Media & $\mathrm{DE}$ & Media & $\mathrm{DE}$ & Media & $\mathrm{DE}$ & Media & $\mathrm{DE}$ & Media & $\mathrm{DE}$ & Media & $\mathrm{DE}$ \\
\hline Involucramiento parental & 2.1 & 0.9 & 2.4 & 0.8 & 2.3 & 0.8 & 2.0 & 0.9 & 2.5 & 0.8 & 2.3 & 0.9 \\
\hline Monitoreo negativo & 2.1 & 0.9 & 2.5 & 0.9 & 2.5 & 0.9 & 1.7 & 0.8 & 2.2 & 0.9 & 2.1 & 0.9 \\
\hline Aliento parental & 2.4 & 0.9 & 2.6 & 0.9 & 2.5 & 0.9 & 2.2 & 1.0 & 2.7 & 1.0 & 2.5 & 1.0 \\
\hline Disciplina inconsistente & 2.1 & 0.8 & 2.2 & 0.8 & 2.1 & 0.8 & 2.0 & 0.8 & 2.2 & 0.8 & 2.1 & 0.8 \\
\hline Amigos prosociales & 2.2 & 0.6 & 2.4 & 0.7 & 2.3 & 0.6 & 2.2 & 0.6 & 2.5 & 0.6 & 2.4 & 0.6 \\
\hline Amigos antisociales & 1.7 & 0.7 & 2.3 & 0.7 & 2.3 & 0.7 & 1.7 & 0.6 & 2.3 & 0.6 & 2.3 & 0.6 \\
\hline $\begin{array}{l}\text { Percepción de riesgo de } \\
\text { uso de drogas ilícitas }\end{array}$ & 1.3 & 0.5 & 1.8 & 0.6 & 1.6 & 0.6 & 1.2 & 0.5 & 1.8 & 0.6 & 1.6 & 0.6 \\
\hline $\begin{array}{l}\text { Percepción de riesgo de } \\
\text { uso de drogas legales }\end{array}$ & 1.4 & 0.6 & 1.8 & 0.7 & 1.7 & 0.6 & 1.4 & 0.5 & 1.8 & 0.6 & 1.7 & 0.6 \\
\hline
\end{tabular}


Tabla 5

Factores de riesgo de uso de inhalables comparados con uso de otras drogas y no consumo de drogas por sexo

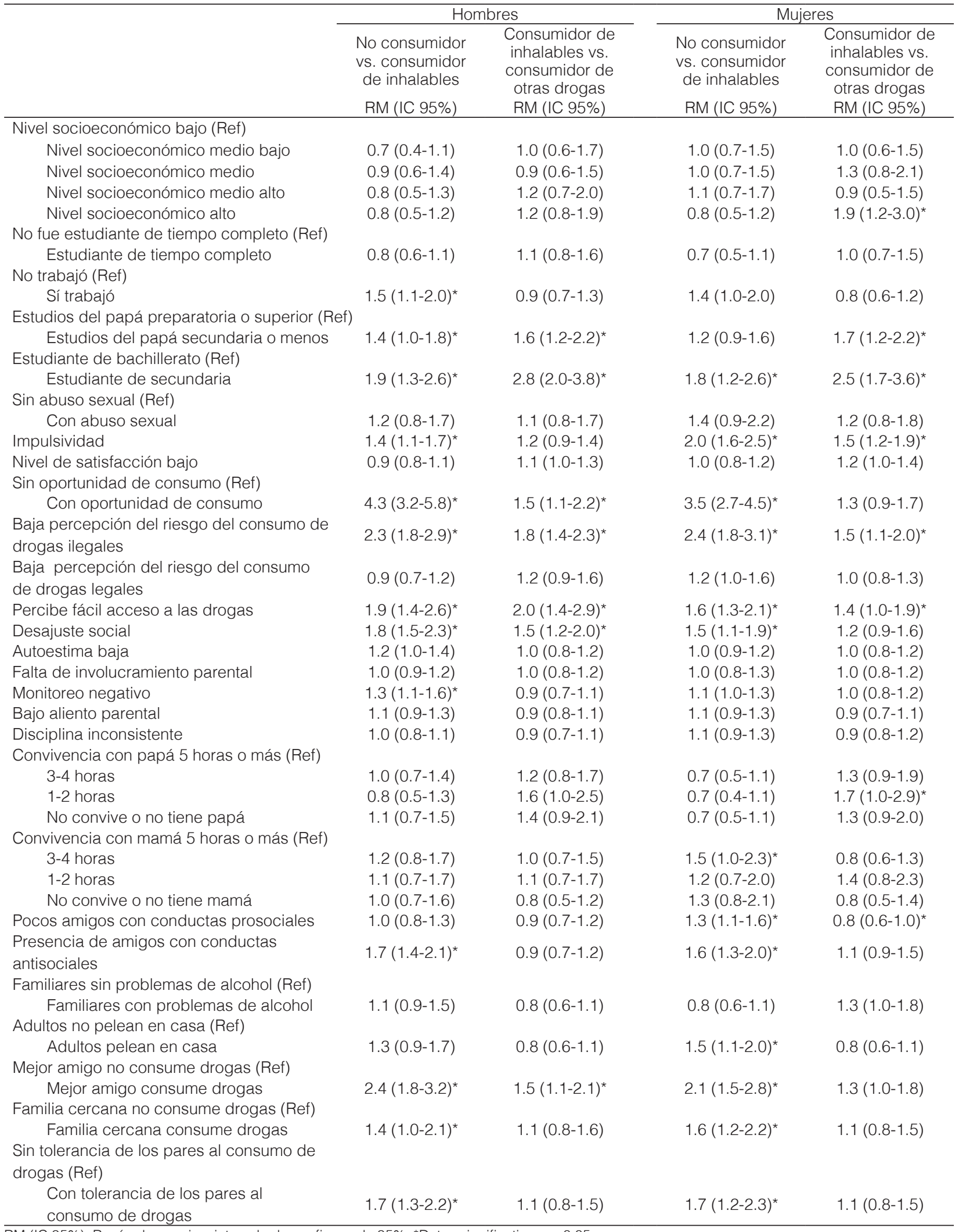

RM (IC 95\%): Razón de momios, intervalo de confianza de 95\%. *Datos significativos $p<0.05$. 
daria, presentan una percepción baja de riesgo de uso de drogas ilegales y tienen un acceso fácil a las mismas, comparados con no usuarios o con consumidores de otras sustancias. Asimismo, los usuarios de inhalables presentan mayor impulsividad, más amigos con problemas antisociales, tienen algún familiar consumidor de drogas y se encuentran en un contexto social en el que los pares toleran el uso de sustancias.

Esta información complementa lo encontrado en poblaciones vulnerables, como los niños que viven en situación de indigencia (Gigengack, 2014a, 2014c, 2014d). Es importante ver que tanto en las poblaciones en situación de calle como entre los estudiantes, algunas de las características más importantes son la disponibilidad y la oportunidad de consumo. Para poder abordar esto, además de crear estrategias psicosociales, es necesario proveer de herramientas y habilidades sociales a los niños y adolescentes que les permitan afrontar el contexto en el que interactúan. Si bien es poco probable que los adolescentes cambien su entorno, es necesario que aprendan a afrontar la situación familiar y social en la que se desenvuelven.

A pesar de que los inhalables son productos industriales muy potentes, los estudiantes tienden a creer que no son tan dañinos como otras drogas. Es posible que además de su fácil acceso, también esta baja percepción de daño esté relacionada con su uso; por lo tanto, es necesaria una mayor información que explique los daños físicos y las conductas de riesgo relacionadas con el uso de inhalables. Esto se puede hacer a través de programas de difusión como los que ya se han implementado a través de la Secretaría de Salud del Gobierno del Distrito Federal o los que son promovidos por centros de tratamiento. Es fundamental incluir a las escuelas en dichos programas, pues la antigua percepción de que los inhalables son únicamente usados por niños y adolescentes que no están dentro del sistema escolar no es correcta y este artículo lo muestra.

También es importante destacar que las personas que consumen otras drogas diferentes a los inhalables reportaron significativamente mayor oportunidad de uso de drogas. Por esto, es importante pensar en programas integrales. Los consumidores de otras drogas, además de estar en un contexto posiblemente más vulnerable, también pueden estar en mayor riesgo de incidir en conductas ilícitas para conseguir las sustancias que usan.

\section{FUENTES DE FINANCIAMIENTO}

Este proyecto recibió financiamiento de la Administración Federal de los Servicios Educativos para el Distrito Federal y del Instituto Nacional de Psiquiatría Ramón de la Fuente Muñiz.

\section{CONFLICTOS DE INTERÉS}

Los autores de este artículo declaramos que no existe ningún conflicto de interés en la realización de este manuscrito.

\section{AGRADECIMIENTOS}

A la Administración Federal de los Servicios Educativos para el Distrito Federal, por su apoyo y disponibilidad para realizar el proyecto en cuestión.

\section{REFERENCIAS}

Balster, R. L., Cruz, S., Howard, M. O., Dell, C. A., \& Cottler, L. B. (2008). Classification of abused inhalants. Addiction, 104, 878-882.

Cruz, S. (2011). El abuso de inhalables: problema creciente de salud publica. Avance y Perspectiva, 3(3), 2-11.

Degenhardt, L., Chiu, W. T., Sampson, N., Kessler, R. C., Anthony, J. C., Angermeyer, M., ... Wells, J. E. (2008). Toward a global view of alcohol, tobacco, cannabis, and cocaine use: findings from the WHO World Mental Health Surveys. PLoS Medicine, 5(7), e141.

Gigengack, R. (2014a). La banda y sus choros. Un grupo de niños de la calle hilando historias de edad, genero y liderazgo. Salud Mental, 37, 329-339.

Gigengack, R. (2014b). "My body breaks. I take solution". Inhalant use in Delhi as pleasure seeking at a cost. International Journal of Drug Policy, 25, 810-818.

Gigengack, R. (2014c). The badass and the asshole: violence and the positioned subjectivities of street youth in Mexico City. In $\mathrm{H}$. Moksnes \& M. Melin (Eds.), Violence and Urban Politics (pp. 172-184). The Netherlands: Uppsala University.

Gigengack, R. (2014d). The chemo and the mona: inhalants, devotion and street youth in Mexico City. International Journal of Drug Policy, 25, 61-70.

Instituto Nacional de Psiquiatría Ramón de la Fuente Muñiz, Instituto Nacional de Salud Publica, \& Secretaria de Salud. (2012). Encuesta Nacional de Adicciones 2011: Reporte de Drogas. Villatoro, JA, Medina-Mora ME, Fleiz C, Tellez-Rojo MM, Mendoza LR, Romero M, Gutiérrez JP, Castro-Tinoco M, Hernández-Avila M, Tena-Tamayo C, Alvear-Sevilla C y Guisa-Cruz V. México, DF, México: INPRFM Disponible en: http://www.inprf.gob.mx, http://www.conadic.gob.mx, http://www.cenadic.salud.gob.mx, http://www.insp.gob.mx.

Johnston, L. D., O’Malley, P. M., Miech, R. A., Bachman, J. G., \& Schulenberg, J. E. (2015). Monitoring the Future national results on drug use: 1975-2014: Overview, Key Findings on Adolescent Drug Use. Ann Arbor: Institute for Social Research, The University of Michigan.

Maclver, M. B. (2009). Abused inhalants enhance GABA-mediated synaptic inhibition. Neuropsychopharmacology, 34, 22962304. 
Medina-Mora, M. E., Villatoro, J., Fleiz, C., Dominguez, M., \& Cruz, S. (2014). Challenges to neuroscience and public policy derived from new trends and patterns of inhalant misuse. Journal of Drug and Alcohol Research, 3(1-8).

Morris, M. D., Case, P., Robertson, A. M., Lozada, R., Vera, A., Clapp, J. D., ... Strathdee, S. A. (2011). Prevalence and correlates of 'agua celeste'use among female sex workers who inject drugs in Ciudad Juarez, Mexico. Drug and Alcohol Dependence, 117(2), 219-225.

Nonnemaker, J. M., Crankshaw, E. C., Shive, D. R., Hussin, A. H., \& Farrelly, M. C. (2011). Inhalant use initiation among US adolescents: evidence from the National Survey of Parents and Youth using discrete-time survival analysis. Addictive Behaviors, 36, 878-881.

Observatorio Europeo de las Drogas y las Toxicomanías (2015). Informe Europeo sobre Drogas. Tendencias y novedades. Luxemburgo: Autor

Rosenberg, N. L., Spitz, M. C., Filley, C. M., Davis, K. A., \& Schaumburg, H. H. (1988). Central nervous system effects of chronic toluene abuse- clinical, brainstem evoked response and magnetic resonance imaging studies. Neurotoxicology and Teratology, 10, 489-495.

Schepis, T. S. \& Krishnan-Sarin, S. (2008). Characterizing adolescent prescription misusers: a population-based study. Journal of American Academia of Child and Adolescent Psychiatry, 47(7), 745-754.

Siegel, J. T., Alvaro, E. M., Patel, N., \& Crano, W. D. (2009). “... you would probably want to do it. Cause that's what made them popular": exploring perceptions of inhalant utility among young adolescent nonusers and occasional users. Substance Use \& Misuse, 44, 597-615.

Storr, C. L., Westergaard, R., \& Anthony, J. C. (2005). Early onset inhalant use and risk for opiate initiation by young adulthood. Drug and Alcohol Dependence, 78, 253-261.
Takagi, M. J., Lubman, D. I., \& Yucel, M. (2007). Interpreting neuropsychological impairment among adolescent inhalant users: two case reports. Acta Neuropsychiatrica, 20, 41-43.

Toga, A. W., Thompson, P. M., \& Sowell, E. R. (2006). Mapping brain maturation. Trends in Neuroscience, 29, 148-159.

Vaughn, M. G., Perron, B. E., \& Howard, M. O. (2007). Variations in social contexts and their effect on adolescent inhalant use: a latent profile investigation. Drug and Alcohol Dependence, 91, 129-133.

Villatoro, J., Cruz, S., Ortiz, A., \& Medina-Mora, M. E. (2011). Volatile substance misuse in Mexico: correlates and trends. Substance Use \& Misuse, 46, 40-45.

Villatoro, J. A., Gaytán, F., Moreno, M., Gutiérrez, M. D. L., Oliva, N., Bretón, M., ... Blanco, C. (2011). Tendencias del uso de drogas en la Ciudad de México: Encuesta de Estudiantes del 2009. Salud Mental, 34(2), 81-94.

Villatoro, J. A., Gutiérrez, M. D. L., Quiroz, N., Moreno, M., Gaytán, L., Gaytán, F. I., ... Elena, M. (2009). Encuesta de estudiantes de la Ciudad de México 2006: Prevalencias y evolución del consumo de drogas. Salud Mental, 32(4), 287-297.

Wu, L. T., Schlenger, W. E., \& Ringwalt, C. L. (2005). Use of nitrate inhalants ("poppers") among American youth. Journal of Adolescent Health, 37, 52-60.

Yucel, M., Takagi, M., Walterfang, M., \& Lubman, D. I. (2008). Toluene misuse and long-term harms: a systematic review of the neuropsychological and neuroimaging literature. Neuroscience and Biobehavioral Reviews, 32, 910-926.

Yücel, M., Zalesky, A., Takagi, M. J., Bora, E., Fornito, A., Ditchfield, M., ... Lubman, D. I. (2010). White-matter abnormalities in adolescents with long-term inhalant and cannabis use: a diffusion magnetic resonance imaging study. Journal of Psychiatry \& Neuroscience, 35(6), 409 